\title{
Présence du glouton Gulo gulo (Mustelidae, Carnivora) dans le gisement pléistocène moyen de La Fage-Aven II, à Noailles (Corrèze, France)
}

The presence of wolverine Gulo gulo (Mustelidae, Carnivora) in the middle Pleistocene settlement of La Fage-Aven II, in Noailles (Corrèze, France)

Gaëtan Bourgeois et Michel Philippe

\section{OpenEdition}

Journals

Édition électronique

URL : http://journals.openedition.org/paleo/3479

DOI : $10.4000 /$ paleo.3479

ISSN : $2101-0420$

Éditeur

SAMRA

Édition imprimée

Date de publication : 30 décembre 2017

Pagination : 215-226

ISSN : $1145-3370$

Référence électronique

Gaëtan Bourgeois et Michel Philippe, «Présence du glouton Gulo gulo (Mustelidae, Carnivora) dans le gisement pléistocène moyen de La Fage-Aven II, à Noailles (Corrèze, France) », PALEO [En ligne], 28 | 2017, mis en ligne le 01 juin 2018, consulté le 07 juillet 2020. URL : http://journals.openedition.org/ paleo/3479 ; DOI : https://doi.org/10.4000/paleo.3479

\section{(c) (i) $\Theta$}

PALEO est mis à disposition selon les termes de la licence Creative Commons Attribution - Pas d'Utilisation Commerciale - Pas de Modification 4.0 International. 
Paleo XXVIII Paleo XXVIII Paleo XXVIII Paleo XXVIII Paleo XXVIII Paleo XXVIII Paleo XXVIII Paleo XXVIII Paleo XXVIII Paleo XXVIII Paleo XXVIII Paleo XXVIII Paleo XXVIII Paleo XXVIII Paleo XXVIII Paleo XXVIII Paleo XXVIII Paleo XXVIII P Paleo XXVIII Paleo XXVIII Paleo XXVIII Paleo XXVIII P Paleo XXVIII Paleo XXVIII Paleo XXVIII Paleo XXVIII P Paleo XXVIII Paleo XXVIII Paleo XXVIII Paleo XXVIII P Paleo XXVIII Paleo XXVIII Paleo XXVIII Paleo XXVIII P Paleo XXVIII Paleo XXVIII Paleo XXVIII Paleo XXVIII P Paleo XXVIII Paleo XXVIII Paleo XXVIII Paleo XXVIII P Paleo XXVIII Paleo XXVIII Paleo XXVIII Paleo XXVIII P Paleo XXVIII Paleo XXVIII Paleo XXVIII Paleo XXVIII P Paleo XXVIII Paleo XXVIII Paleo XXVIII Paleo XXVIII P Paleo XXVIII Paleo XXVIII Paleo XXVIII Paleo XXVIII P Paleo XXVIII Paleo XXVIII Paleo XXVIII Paleo XXVIII P Paleo XXVIII Paleo XXVIII Paleo XXVIII Paleo XXVIII P Paleo XXVIII Paleo XXVIII Paleo XXVIII Paleo XXVIII P Paleo XXVIII Paleo XXVIII Paleo XXVIII Paleo XXVIII P Paleo XXVIII Paleo XXVIII Paleo XXVIII Paleo XXVIII P Paleo XXVIII Paleo XXVIII Paleo XXVIII Paleo XXVIII P Paleo XXVIII Paleo XXVIII Paleo XXVIII Paleo XXVIII P

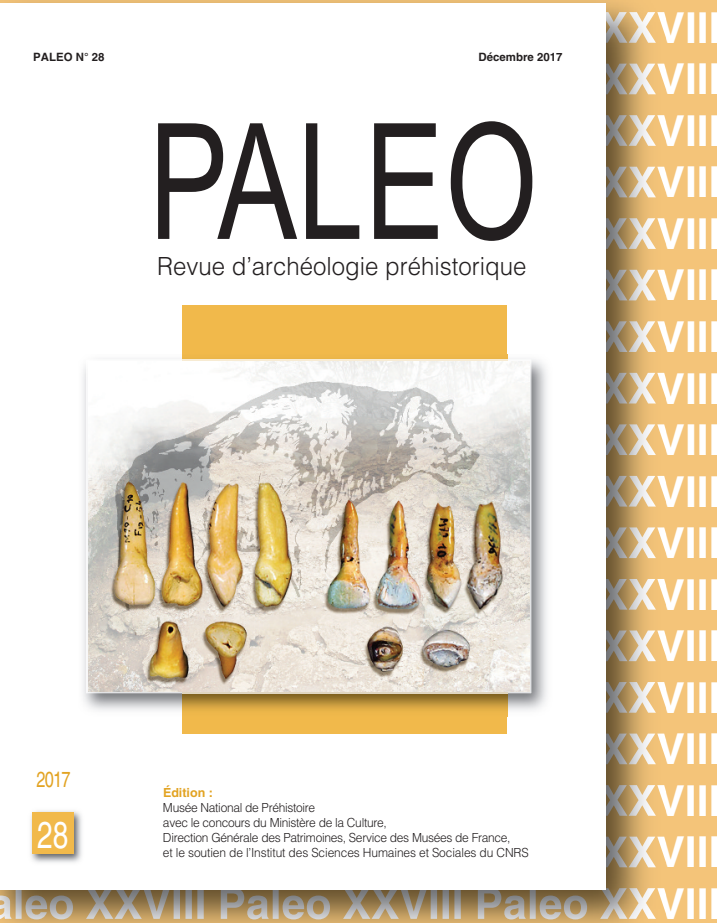
ш Paleo XXVIII Paleo XXVIII Paleo XXVIII Paleo XXVIII Paleo XXVIII Paleo XXVIII Paleo XXVIII Paleo XXVIII Paleo XXVIII Paleo XXVIII Paleo XXVIII Paleo XXVIII Paleo XXVIII Paleo XXVIII Paleo XXVIII Paleo XXVIII Paleo XXVIII Paleo XXVIII Paleo XXVIII Paleo XXVIII Paleo XXVIII Paleo XXVIII Paleo XXVIII Paleo XXVIII Paleo XXVIII Paleo XXVIII Paleo XXVIII Paleo XXVIII Paleo XXVIII Paleo XXVIII Paleo XXVIII Paleo XXVIII Paleo XXVIII Paleo XXVIII Paleo XXVIII Paleo XXVIII Paleo XXVIII Paleo XXVIII Paleo XXVIII Paleo XXVIII Paleo XXVIII Paleo XXVIII Paleo XXVIII Paleo XXVIII Paleo XXVIII Paleo XXVIII Paleo XXVIII Paleo XXVIII Paleo XXVIII Paleo XXVIII Paleo XXVIII Paleo XXVIII Paleo XXVIII Paleo XXVIII Paleo XXVIII Paleo XXVIII Paleo XXVIII Paleo XXVIII Paleo XXVIII Paleo XXVIII Paleo XXVIII Paleo XXVIII Paleo XXVIII Paleo XXVIII Paleo XXVIII Paleo XXVIII Paleo XXVIII Paleo XXVIII Paleo XXVIII Paleo XXVIII Paleo XXVIII Paleo XXVIII Paleo XXVIII Paleo XXVIII Paleo XXVIII Paleo XXVIII Paleo XXVIII Paleo XXVIII Paleo XXVIII Paleo XXVIII Paleo XXVIII Paleo XXVIII Paleo XXVIII Paleo XXVIII Paleo XXVIII Paleo XXVIII Paleo XXVIII Paleo XXVIII Paleo XXVIII Paleo XXVIII Paleo XXVIII Paleo XXVIII Paleo XXVIII Paleo XXVIII Paleo XXVIII Paleo XXVIII Paleo XXVIII Paleo XXVIII Paleo XXVIII Paleo XXVIII Paleo XXVIII Paleo XXVIII Paleo XXVIII Paleo XXVIII Paleo XXVIII Paleo XXVIII Paleo XXVIII Paleo XXVIII Paleo XXVIII Paleo XXVIII Paleo XXVIII Paleo XXVIII Paleo XXVIII Paleo XXVIII Paleo XXVIII Paleo XXVIII Paleo XXVIII Paleo XXVIII Paleo XXVIII Paleo XXVIII Paleo XXVIII Paleo XXVIII Paleo XXVIII Paleo XXVIII Paleo XXVIII Paleo XXVIII Paleo XXVIII Paleo XXVIII Paleo XXVIII Paleo XXVIII Paleo XXVIII Paleo XXVIII Paleo XXVIII Paleo XXVIII Paleo XXVIII Paleo XXVIII Paleo XXVIII Paleo XXVIII Paleo XXVIII Paleo XXVIII Paleo XXVIII Paleo XXVIII Paleo XXVIII Paleo XXVIII Paleo XXVIII Paleo XXVIII Paleo XXVIII Paleo XXVIII Paleo XXVIII Paleo XXVIII Paleo XXVIII Paleo XXVIII Paleo XXVIII Paleo XXVIII Paleo XXVIII Paleo XXVIII Paleo XXVIII Paleo XXVIII Paleo XXVIII Paleo XXVIII Paleo XXVIII Paleo XXVIII Paleo XXVIII Paleo XXVIII Paleo XXVIII Paleo XXVIII Paleo XXVIII Paleo XXVIII Paleo XXVIII Paleo XXVIII Paleo XXVIII Paleo XXVIII Paleo XXVIII Paleo XXVIII Paleo XXVIII Paleo XXVIII Paleo XXVIII Paleo XXVIII Paleo XXVIII Paleo XXVIII Paleo XXVIII Paleo XXVIII Paleo XXVIII Paleo XXVIII Paleo XXVIII Paleo XXVIII Paleo XXVIII Paleo XXVIII Paleo XXVIII Paleo XXVIII Paleo XXVIII Paleo XXVIII Paleo XXVIII Paleo XXVIII Paleo XXVIII Paleo XXVIII Paleo XXVIII Paleo XXVIII Paleo XXVIII Paleo XXVIII Paleo XXVIII Paleo XXVIII Paleo XXVIII Paleo XXVIII Paleo XXVIII Paleo XXVIII Paleo XXVIII Paleo XXVIII Paleo XXVIII Paleo XXVIII Paleo XXVIII Paleo XXVIII Paleo XXVIII Paleo XXVIII Paleo XXVIII Paleo XXVIII Paleo XXVIII Paleo XXVIII Paleo XXVIII Paleo XXVIII Paleo XXVIII Paleo XXVIII Paleo XXVIII Paleo XXVIII Paleo XXVIII Paleo XXVIII Paleo XXVIII Paleo XXVIII Paleo XXVIII Paleo XXVIII Paleo XXVIII Paleo XXVIII Paleo XXVIII Paleo XXVIII Paleo XXVIII Paleo XXVIII Paleo XXVIII Paleo XXVIII Paleo XXVIII Paleo XXVIII Paleo XXVIII Paleo XXVIII Paleo XXVIII Paleo XXVIII Paleo XXVIII 



\title{
Présence du glouton Gulo gulo (Mustelidae, Carnivora) dans le gisement pléistocène moyen de La Fage-Aven II, à Noailles (Corrèze, France)
}

\author{
Gaëtan BOURGEOIS ${ }^{(a)}$, Michel PHILIPPE ${ }^{(b)}$
}

\begin{abstract}
Résumé : Nous présentons dans cette étude une ulna de glouton (Gulo gulo) venant s'ajouter à la liste faunique du gisement pléistocène moyen de l'Aven II des Abîmes de La Fage (Corrèze). Sa présence, sous cette latitude, indique que le climat était alors beaucoup plus froid qu'aujourd'hui. Elle pourrait remonter à la fin du MIS 6, près du « pic de froid » qui précède de peu la mise en place du sous-stade $5 \mathrm{e}$, voire à une période plus récente. La petite taille du spécimen pourrait être le signe d'une adaptation à des conditions sévères pour l'espèce qui vivait alors en limite de son aire de répartition, conformément aux lois de Bergmann et d'Allen.
\end{abstract}

Mots-clés : Pléistocène moyen, La Fage, Carnivora, Mustelidae, Glouton, Gulo gulo, paléontologie, biométrie, paléoenvironnement.

Abstract: The presence of wolverine Gulo gulo (Mustelidae, Carnivora) in the middle Pleistocene settlement of La Fage-Aven II, in Noailles (Corrèze, France). In this study we present an ulna of wolverine (Gulo gulo), added to the faunal list of the Middle Pleistocene deposit of Aven II of the Abyss of La Fage (Correze). Its presence, under this latitude, indicates that the climate was way much colder than today. It could be dated back to the end of MIS 6, near the " peak of cold » which precedes an establishment of sub-stage $5 \mathrm{e}$ or even to a more recent period. The small size of the specimen could be a sign of adaptation to severe conditions for the species that lived at the limit of its natural range, in accordance with the laws of Bergmann and Allen.

Key-words: Middle Pleistocene, La Fage, Carnivora, Mustelidae, Wolverine, Gulo gulo, palaeontology, biometry, palaeoenvironment.

\section{1 - Introduction et problématique}

Lors d'une étude de la collection fossile de l'Aven II de La Fage, conservée au Centre de Conservation et d'Étude des Collections (rattaché au Musée des Confluences de Lyon), une ulna a attiré notre attention. Jusqu'alors déterminée comme étant un os de blaireau (Meles meles), elle nous a semblé être en réalité un reste de glouton (Gulo gulo).
Le présent travail cherche à vérifier cette détermination et à évaluer les incidences sur notre connaissance du contexte paléoclimatique et paléoenvironnemental de ce gisement. Ce site et notamment sa liste faunique étant par ailleurs déjà connus et décrits (Bouchud 1978 ; Philippe 1978), cette ulna sera l'unique objet étudié ici.

(a) Université de Perpignan Via Domitia, ED 544, Laboratoire d'Histoire Naturelle de l'Homme Préhistorique (HNHP) Centre Européen de Recherche Préhistorique de Tautavel, UMR CNRS 7194 et EPCC - CERPT, Avenue Léon-Jean Grégory, 66720 Tautavel gaetan.bourgeois@cerptautavel.com

(b) Conservateur honoraire de Muséum d'Histoire naturelle de Lyon (actuel Musée des Confluences). Centre de Conservation et d'Étude des Collections (Musée des Confluences), 13A rue Bancel, 69007 Lyon. 


\section{2 - Présentation du site}

L'aven II des " Abîmes de La Fage » se situe à l'extrême nord du Causse de Martel qui constitue la partie la plus septentrionale des causses du Quercy. II s'ouvre sur le territoire de la commune de Noailles (Corrèze), à quelques centaines de mètres seulement de la grande faille qui sépare ce causse jurassique du bassin permo-triasique de Brive (Coordonnées GPS : 454'41.8” Nord et 1³1'28.13" Est, fig. 1).

Comme dans de nombreuses régions calcaires, ce secteur est fortement karstifié et la plupart des cavités sont en grande partie colmatées par des remplissages, souvent fort anciens et parfois fossilifères (Guérin et Philippe 1971 ; Philippe, Mourer-Chauviré et Evin 1981).

L'Aven II n'est distant que d'une vingtaine de mètres du célèbre Aven I et se présente de façon très analogue : un tronçon de galerie effondré dont l'ouverture a été entièrement colmatée (fig. 2).
Il a été fouillé entre juin et novembre 1964 par C. Dayre, propriétaire du terrain et $\mathrm{L}$. Tauran. Cette opération a été entreprise avec l'espoir de découvrir, sinon un autre gisement, du moins des tronçons de galeries susceptibles de venir compléter le circuit touristique du « Gouffre de La Fage ». Les travaux de désobstruction de cet aven furent suivis avec attention par Michel Philippe qui consigna les observations de terrain sur un cahier de fouille, prit part à l'extraction des amas osseux ainsi qu'au prélèvement et au lavage des sédiments.

Bien que la fouille n'ait pas été conduite avec autant de rigueur que ce qui aurait été souhaité, notamment pour le repérage en trois dimensions de chaque vestige osseux, on peut malgré tout considérer que les conditions nécessaires à une connaissance suffisamment précise de la formation du gisement, de la faune recueillie et de sa répartition au sein du remplissage ont été réunies.

La totalité des vestiges osseux recueillis dans ce gisement est conservée dans les collections du Centre de Conservation et d'Étude des Collections rattaché au Musée des Confluences (ex-Muséum d'Histoire naturelle de Lyon).

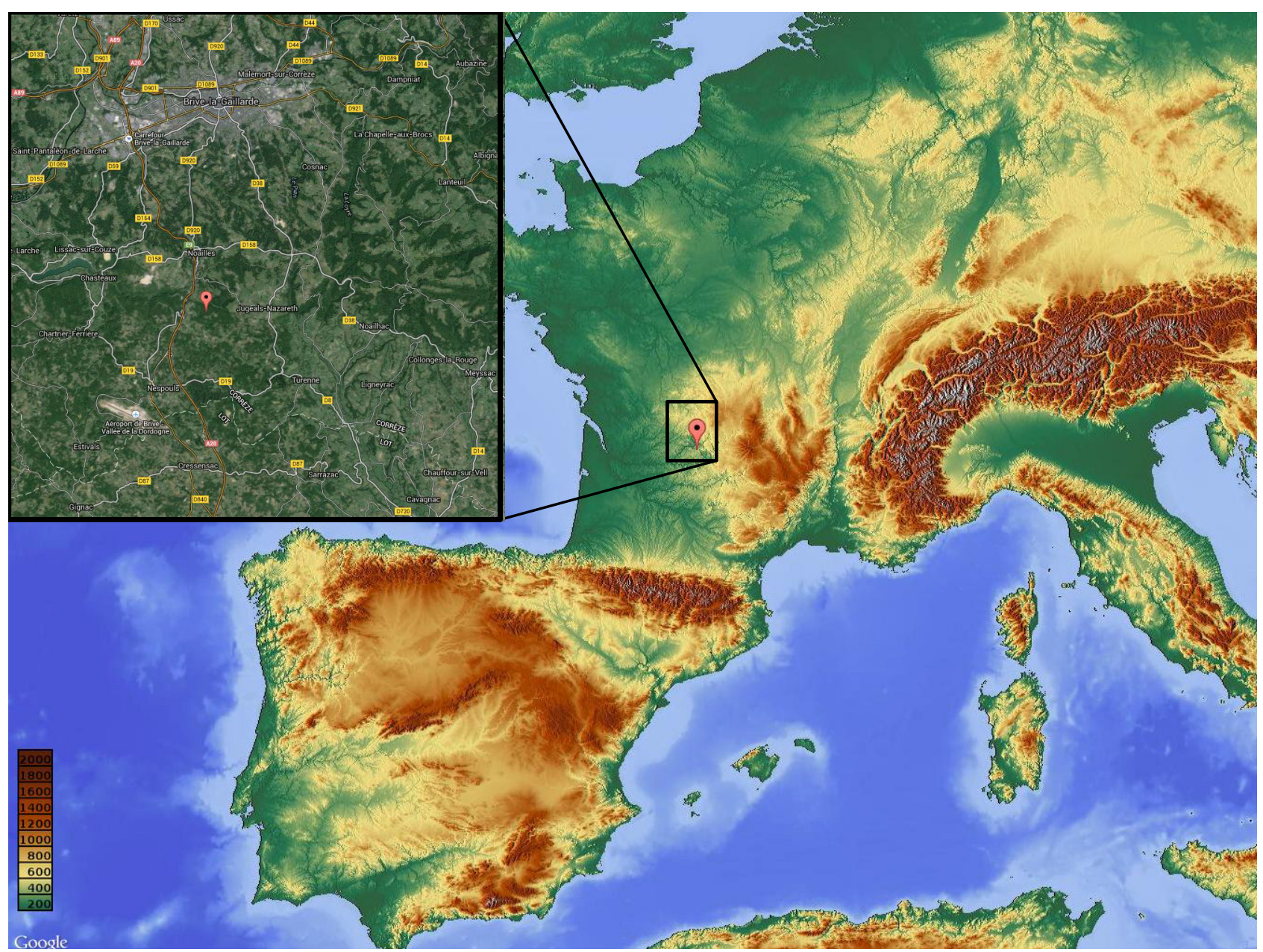

Figure 1 - Carte situant le gisement de La Fage - Aven II.

Figure 1 - Map showing the geographic situation of La Fage - Aven II settlement. 


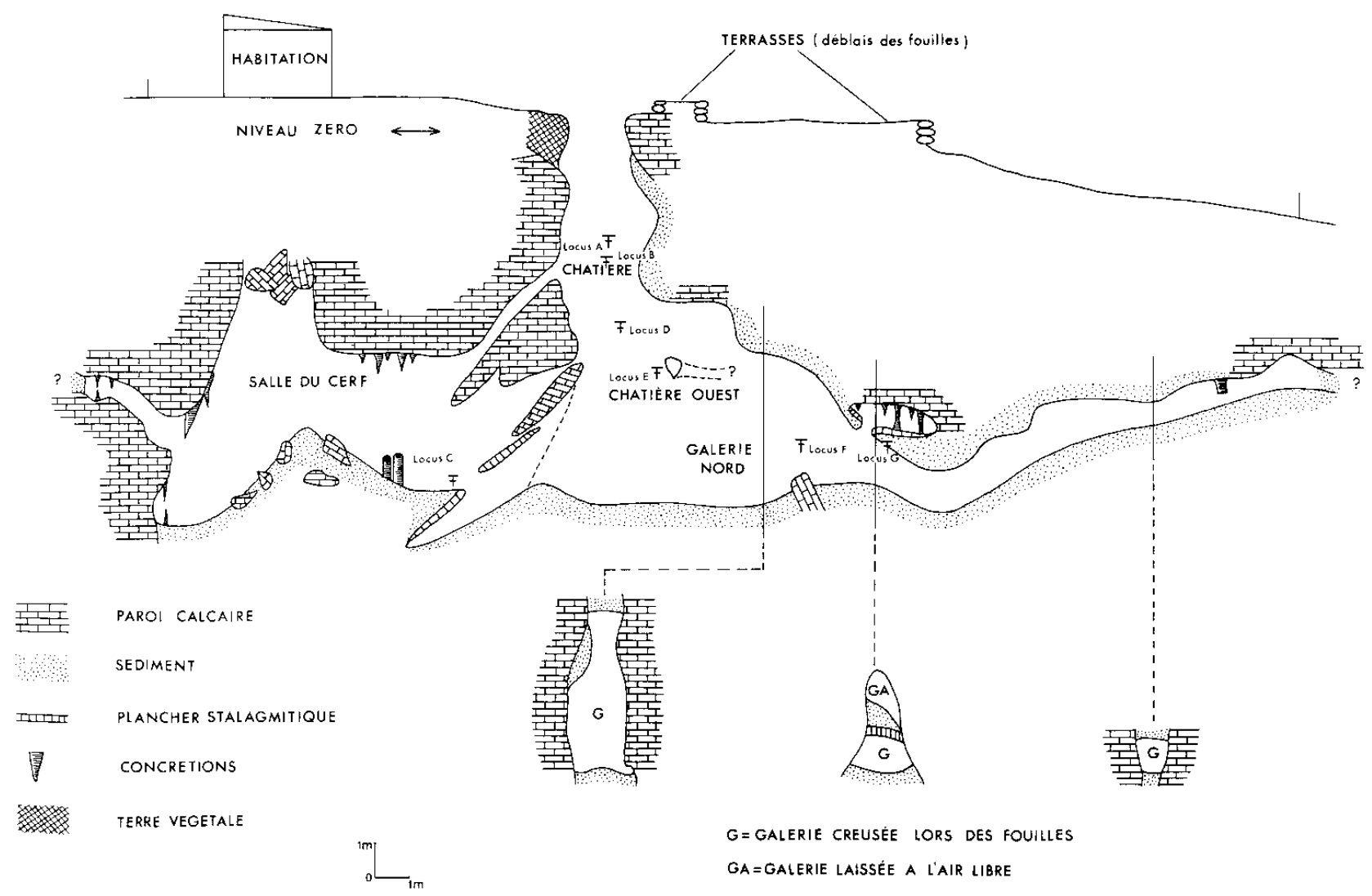

Figure 2 - Coupe schématique de la stratigraphie du gisement de La Fage - Aven II, d'après Philippe 1978.

Figure 2 - Schematic section of the stratigraphy of the site of La Fage - Aven II, after Philippe 1978.

À ce jour, seuls les restes de grands herbivores recueillis dans ce gisement ont été étudiés et décrits par J. Bouchud (1978). Les autres ossements signalés sont du fait de M. Philippe (1978) et du présent travail.

D'environ deux mètres de diamètre, cet aven à l'origine totalement comblé a été vidé sur une dizaine de mètres de profondeur. À mi-hauteur, sur la paroi sud, s'ouvre une petite chatière non obstruée donnant accès à une salle, la salle du Cerf, dont le plafond, fermé par de gros blocs rocheux, correspond à un autre aven latéral à l'Aven II. Vers les 10 mètres de profondeur, on accède à deux tronçons de galerie encore partiellement libres : le tronçon sud rejoint la salle évoquée précédemment et le tronçon nord a été exploré sur une vingtaine de mètres de long, parfois déblayé de sédiments qui l'encombraient pour permettre la progression. Ce travail de désobstruction a été stoppé sur un rétrécissement de la galerie laissant peu d'espoir à de nouvelles découvertes paléontologiques. Contrairement à l'Aven I qui livre des fossiles sur toute l'épaisseur du remplissage, les ossements de l'Aven II étaient essentiellement regroupés en amas. Ils sont désignés sur la coupe de la cavité (fig. 2) par des lettres (locus $\mathrm{A}, \mathrm{B}, \ldots$ ) correspondant à la chronologie des découvertes et peu ou prou à la stratigraphie du gisement. - dans le locus A, dès 3,50 m de profondeur ont été trouvés des fragments d'un crâne, d'une extrémité proximale d'humérus de blaireau et d'une mandibule d'un autre Mustélidé ;

- dans le locus $B$, de -4 à $-4,50 \mathrm{~m}$, reposaient deux fragments crâniens, une canine inférieure droite, un fragment de vertèbre lombaire, une extrémité proximale et une extrémité distale d'humérus peut-être du même animal, trois côtes et une extrémité distale de fémur de blaireau, Meles meles ; une extrémité proximale d'humérus, l'épiphyse distale d'un humérus et la moitié distale d'un autre humérus de chat sauvage, Felis silvestris ; et enfin une hémi-mandibule en deux morceaux mais pratiquement complète d'un petit Mustélidé à rattacher à la fouine, Martes foina, ou à la martre, Martes martes. C'est également de cette lentille que provient l'ulna de glouton ( $n^{\circ} 20100583$ ) faisant l'objet de ce travail. Pour la plupart, les ossements du locus B étaient incomplets, présentant des cassures anciennes et même patinées par la fossilisation, ce qui semble témoigner d'un apport depuis l'extérieur ou d'une remobilisation au sein des galeries, au même titre que les sédiments qui les enveloppaient ; 
- le locus C correspond à la salle du Cerf, vers 10,50 m de profondeur. Là, une cinquantaine d'ossements disposés sur le sol et recouverts de calcite ont été recensés. Outre les vestiges de cerf (Cervus elaphus) qui sont les plus nombreux on peut noter la présence de restes d'élan (Alces alces), de sanglier (Sus scrofa) et d'un peu de microfaune ; - Le locus D a livré 70 restes de cheval appartenant au même individu. J. Bouchud (1978) l'a identifié comme appartenant à la sous-espèce $E$. caballus cf. piveteaui ;

- Le locus E, à -7 m, correspond au départ d'un petit boyau partant vers l'ouest. Le squelette complet d'un jeune blaireau a été trouvé ainsi que de nombreux restes de microfaune ;

- Le locus $\mathrm{F}$ est situé au bas du puits à $-9 \mathrm{~m}$. II a livré d'autres restes de chevaux ainsi qu'un talus de daim (Dama cf. clactoniana), plusieurs os de chevreuil et de léporidé. Les restes de chevaux ont été attribués par J. Bouchud (1978) à Equus caballus cf. steinheimensis;

- enfin, encore plus loin dans la galerie nord, dans le locus $G$, des ossements d'un autre cheval (E. caballus cf. steinheimensis) ont été difficilement extraits d'un plancher stalagmitique sur lequel ils étaient scellés par de la calcite.

Comme dans le cas de l'Aven I, l'Aven II a servi de piège naturel pour des animaux qui y sont vraisemblablement tombés vivants. C'est notamment le cas du cheval pratiquement complet recueilli dans le locus $\mathrm{D}$ et de celui partiellement extrait du locus $\mathrm{G}$ ou encore du blaireau presque complet provenant du locus E. Cependant, Bouchud (1978) n'exclut pas l'hypothèse que certains éléments squelettiques isolés de cervidés (locus F) ou d'équidés (locus G) aient pu arriver dans l'aven lors des phases de sédimentation.

\section{3 - L'ulna de glouton}

\section{1 - Matériel et méthode}

II s'agit d'un fragment d'ulna droite (fig. 3) dont la partie proximale est manquante. La cassure est nette et ancienne, placée juste sous le niveau où devrait débuter la trochlée. Une autre fracture se situe aux deux-tiers de la longueur restante, à partir de l'extrémité distale. Cette dernière semble plus récente, peut-être faite lors de la fouille.

La fossilisation du reste est indéniablement prononcée et semblable aux autres fossiles recueillis dans le même locus. II y a présence sur l'ensemble de l'os de légères fissures de dessiccation et de minuscules cupules de corrosion, en partie recouvertes par des traces d'oxydes de manganèse, qui sont elles-mêmes sous des concrétions ferrugineuses et enfin des concrétions de calcite recouvrant le tout. Cette superposition suggère une période d'exposition aux éléments, avant un enfouissement où se sont succédé les différentes altérations décrites.

L'identification de ce reste osseux a été réalisée par comparaison avec du matériel osseux, actuel et fossile, de gloutons et de blaireaux provenant des collections du Muséum national d'Histoire naturelle de Paris et du Musée

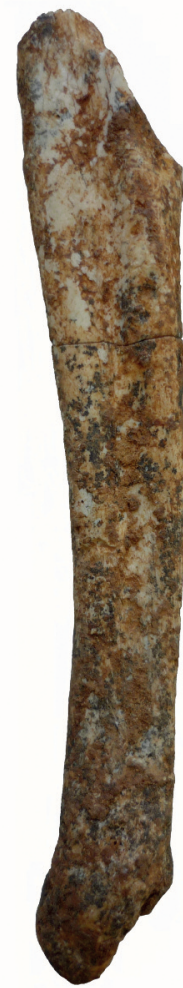

a

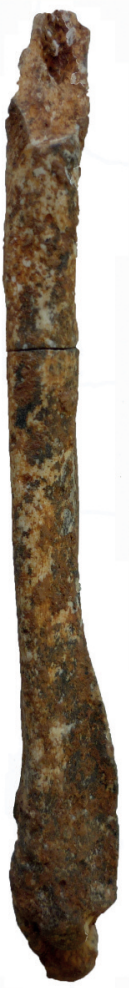

b

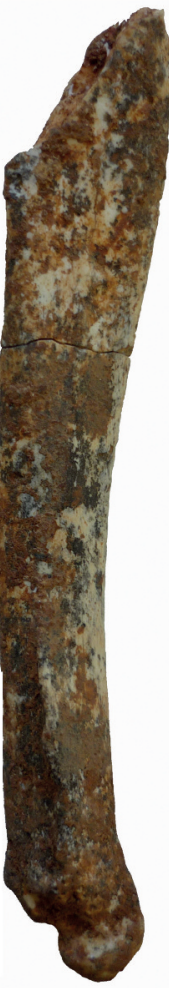

c

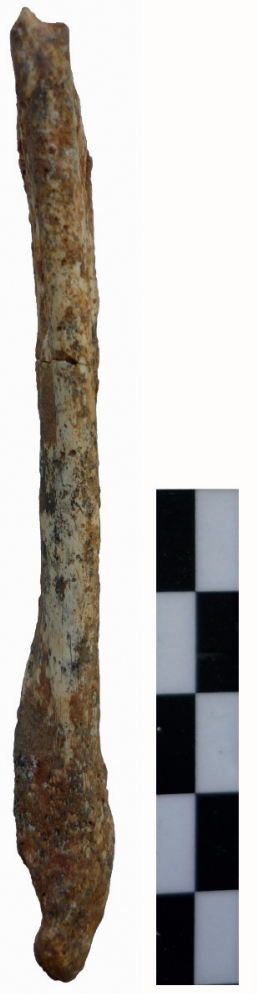

d
Figure 3 - Photos de l'ulna droite de glouton Gulo gulo en vues dorsale (a), palmaire (b), médiale (c) et latérale (d). L'échelle mesure $5 \mathrm{~cm}$ de long (clichés Bourgeois G.).

Figure 3 - Photos of the right ulna of wolverine Gulo gulo in dorsal (a), palmar (b), medial (c) and lateral (d) views. The scale is $5 \mathrm{~cm}$ long (pictures Bourgeois G.).

des Confluences (ex-Muséum d'Histoire naturelle de Lyon), et également à l'aide de planches anatomiques (Lavocat 1966 ; Pales et Lambert 1971) et de photos. Les travaux de Bonifay (1966), Martin (1967, 1968) et Döppes (2001a) concernant la description morphologique des pièces osseuses de glouton et leur distinction avec leurs homologues chez le blaireau ont été pris en considération dans cette étude. Les normes et nomenclatures utilisées sont celles de Barone (1976).

Les mesures que nous avons appliquées à cet os sont adaptées de Von den Driesch (1976) (fig.4). Elles ont été comparées à celles obtenues sur des spécimens de gloutons actuels de comparaison du MNHN (CG 1983-946 et données Mallye et Guérin 2002) et les individus de Villereversure, de la Tanne du Beau-Prince, de la grotte de l'Escale (données Bonifay 1971), de Bouxès (données Crégut-Bonnoure, in Mallye \& Guérin 2002) et de Jaurens (données Mallye et Guérin 2002), conservés au Museum d'Histoire naturelle de Lyon et à l'Université Claude-Bernard (Lyon I) (tabl. 1). Étant donné que le reste est incomplet, la plupart des mesures existant dans la littérature et notamment les longueurs, ne sont malheureusement pas utilisables dans le cadre de cette étude. 


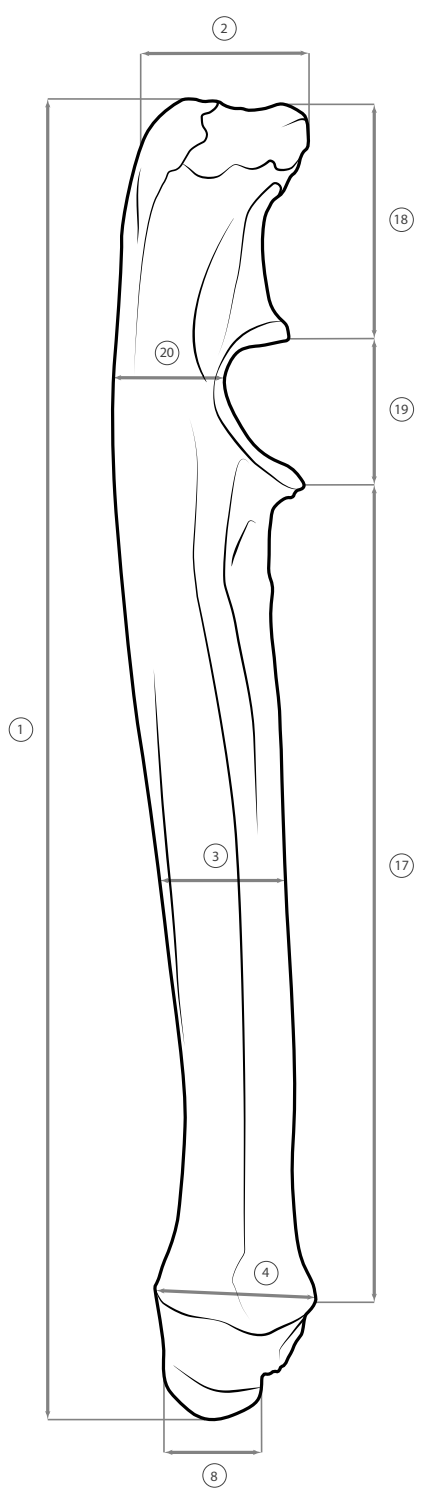

Les morphologies sont différentes entre Gulo et Meles, mais sont tout de même suffisamment proches pour justifier une réserve de prudence. Outre un examen attentif de la morphologie, nous avons utilisé les mesures des ulnas de blaireaux des sites de la grotte du Lazaret, de la Fage-Aven II, d'Ambrussum (spécimens du IVe s. de notre ère, décrits dans Bourgeois 2012) ainsi que les données de Mallye et Guérin (2002) comme groupe extérieur pour réaliser un Log-Size Index (Simpson 1941). Nous avons utilisé la moyenne de ces individus comme référence afin d'observer les différences de proportions (fig. 5).

\section{2 - Résultats}

Les caractéristiques qui permettent de déterminer cette ulna comme se rapportant au glouton tiennent en quatre points :

- en vue latérale (fig. 3a) : le corps ulnaire est plus rectiligne que chez le blaireau ; seul le renflement de la

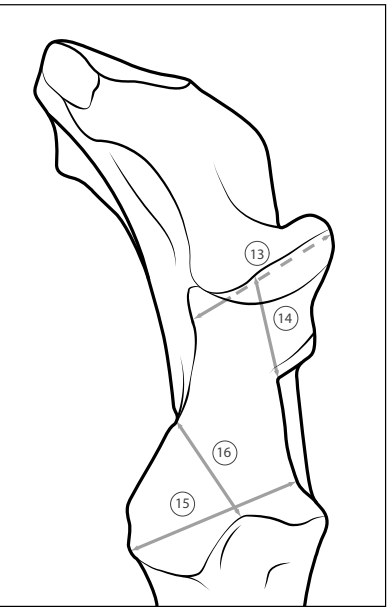

Figure 4 - Schéma de mesurage de l'ulna (dessin Milizia C.).

Figure 4 - Scheme for the measurement of the ulna (drawing Milizia C.).

symphyse distale forme de réelles courbes. En rapport à cela, le processus styloïde est fortement déjeté médialement. ;

- en vue antérieure (fig. 3b) : une épaisseur plus importante, relativement à la longueur. La crête distale est plus développée médialement et convexe. Le processus styloïde est plus trapu. ;

- en vue médiale (fig. 3c) : le corps ulnaire est beaucoup plus incurvé, notamment en sa partie centrale où la concavité dorsale est la plus forte. La crête transverse résultant de la symphyse épiphysaire est peu marquée sur le bord antérieur et pas du tout dorsalement : elle y est au contraire presque concave. Le processus styloïde est anguleux. La partie proximale est très aplatie. Le bord antérieur de la crête distale est fortement creusé par une gouttière. ;

- en vue postérieure (fig. 3d) : le bord distal externe de l'incisure trochléaire est peu marqué. La gouttière latérale est centrée, profonde vers la partie proximale et va en 


\begin{tabular}{|c|c|c|c|c|c|c|c|}
\hline & & 3 & 4 & 6 & 7 & 8 & 9 \\
\hline Individu \mesures & type os & DDP médian & $\begin{array}{l}\text { DDP crête } \\
\text { transverse }\end{array}$ & DT médian & $\begin{array}{l}\text { DT crête } \\
\text { transverse }\end{array}$ & $\begin{array}{c}\text { DDP } \\
\text { processus } \\
\text { styloïde }\end{array}$ & $\begin{array}{l}\text { DT processus } \\
\text { styloïde }\end{array}$ \\
\hline \multicolumn{8}{|l|}{ Meles meles fossiles } \\
\hline Moy. Meles meles Fossiles & Ulnas & 10,04 & 11,14 & 12,01 & 5,65 & 7,82 & 4,87 \\
\hline n Meles meles Fossiles & Ulnas & 7 & 6 & 7 & 7 & 4 & 4 \\
\hline Min. Meles meles Fossiles & Ulnas & 9,3 & 8,45 & 10,89 & 4,545 & 7,37 & 4,63 \\
\hline Max. Meles meles Fossiles & Ulnas & 10,98 & 15,19 & 12,83 & 6,6 & 8,45 & 5,05 \\
\hline S Meles meles Fossiles & Ulnas & 0,495 & 2,958 & 0,705 & 0,809 & 0,526 & 0,175 \\
\hline \multicolumn{8}{|l|}{ Meles meles Actuels } \\
\hline Moy. Meles meles Actuels (Mallye \& Guérin 2002) & Ulnas & 12,6 & 13,3 & 5,6 & 10,2 & & \\
\hline n Meles meles Actuels (Mallye \& Guérin 2002) & Ulnas & 20 & 19 & 20 & 19 & & \\
\hline Min. Meles meles Actuels (Mallye \& Guérin 2002) & Ulnas & 9,9 & 12,3 & 4,5 & 8,4 & & \\
\hline Max. Meles meles Actuels (Mallye \& Guérin 2002) & Ulnas & 14,9 & 14,4 & 6,6 & 11,4 & & \\
\hline S Meles meles Actuels (Mallye \& Guérin 2002) & Ulnas & 1,064 & 0,598 & 0,485 & 0,758 & & \\
\hline \multicolumn{8}{|l|}{ Gulo gulo fossiles } \\
\hline Moy. Gulo gulo fossiles & Ulnas & 12,49 & 17,02 & 7,49 & 13,61 & & \\
\hline n Gulo gulo fossiles & Ulnas & 8 & 7 & 8 & 7 & & \\
\hline Min. Gulo gulo fossiles & Ulnas & 14 & 19 & 8,5 & 15,2 & & \\
\hline Max. Gulo gulo fossiles & Ulnas & 10,4 & 12,93 & 6,02 & 11,6 & & \\
\hline S Gulo gulo fossiles & Ulnas & 1,34 & 2,385 & 0,948 & 1,289 & & \\
\hline \multicolumn{8}{|l|}{ Gulo gulo actuels } \\
\hline Moy. Gulo gulo Actuels & Ulnas & 10,62 & 14,7 & 6,12 & 12,38 & 10,3 & 5,35 \\
\hline n Gulo gulo Actuels & Ulnas & 7 & 7 & 7 & 7 & 2 & 2 \\
\hline Min. Gulo gulo Actuels & Ulnas & 9,48 & 12,33 & 5,32 & 11,2 & 10,17 & 5,28 \\
\hline Max. Gulo gulo Actuels & Ulnas & 12,4 & 18,3 & 7 & 14,5 & 10,42 & 5,41 \\
\hline S Gulo gulo Actuels & Ulnas & 1,23 & 2,39 & 0,719 & 1,341 & 0,177 & 0,092 \\
\hline \multicolumn{8}{|l|}{ G. gulo schlosseri l'Escale (Bonifay, 1971) } \\
\hline G. gulo schlosseri l'Escale H-7377 & Ulna g & & 15,2 & & & & \\
\hline \multicolumn{8}{|l|}{ Gulo gulo La Fage-Aven II } \\
\hline G. gulo La Fage-Aven II 20.100 .583 & Ulna d & 9,34 & 10,34 & 5,02 & 9,04 & 7,11 & 5,06 \\
\hline
\end{tabular}

Tableau 1 - Tableau de mesures des spécimens utilisés pour cette étude.

Table 1 - Table of measures of specimens used in this study.

s'amenuisant progressivement vers la partie distale.

En ce qui concerne l'étude biométrique, seules les mesures 3, 4, 6, 7, 8 et 9 (respectivement Diamètres DorsoPalmaires médian et au niveau de la crête transverse, Diamètres Transverses aux mêmes niveaux, et enfin DDP puis DT au niveau du processus styloïde) sont exploitables pour effectuer les comparaisons. De fait, il n'y a qu'une mesure comparable avec l'individu de la grotte de l'Escale, c'est pourquoi il a été exclu de cette analyse.

Les mesures de l'individu de l'Aven II des Abîmes de La Fage comparées aux moyennes observées chez les gloutons et les blaireaux pris comme référence indiquent que celui-ci suit globalement la tendance de ses congénères, en étant toutefois plus petit. La mesure la plus discriminante entre blaireaux et gloutons est le Diamètre Transverse médian. Sur les mesures considérées ici, ces deux genres affichent une courbe semblable, les gloutons surexprimant toutefois l'écart entre les mesures 4,6 et 7 (DDP crête transverse, DT médian et DT crête transverse). Le spécimen étudié ici présente un DDP au niveau du processus styloïde (mesure 8) moins important, relativement à la moyenne des gloutons actuels.

\section{4 - Chronologie et environnement}

Concernant la position du gisement dans la chronologie du Quaternaire, comme l'a fait remarquer J. Bouchud (1978), les deux sites Aven I et Aven Il étant voisins, leur géométrie et le type de dépôts tout à fait comparables, nous pouvons considérer qu'ils sont très probablement synchrones, au moins partiellement.

En ce qui concerne l'Aven I, la comparaison de la faune qu'il a livré avec celles représentées dans d'autres localités européennes replacées dans la chronologie ont conduit à dater le dépôt de la plupart des niveaux fossilifères des stades isotopiques 8 et 7 (Mourer et al. 2003) : un plancher stalagmitique qui scellait ces dépôts, daté par U/Th, a donné un âge de $291 \mathrm{ka}+\infty$ - 119 ka (cf. fig. 3 de l'article Mourer et al. 2003). Le synchronisme, au moins partiel, de l'Aven II avec l'Aven I semble bien confirmé par la 


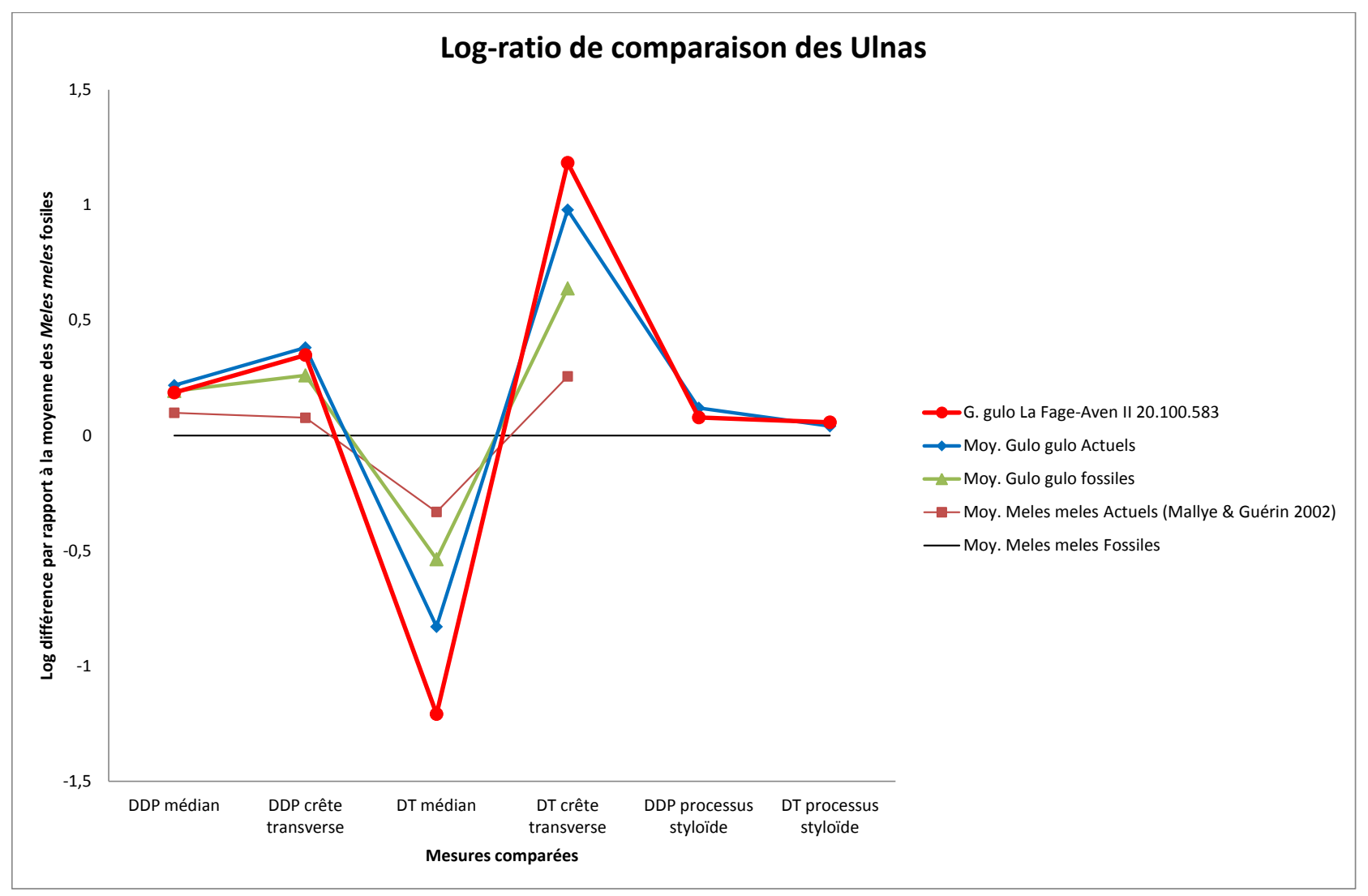

Figure 5 - Diagramme de Log-différence comparant les mesures de l'individu de La Fage - Aven Il à celles de différents spécimens de gloutons et de blaireaux actuels et fossiles.

Figure 5 - Log-ratio diagram comparing the measurements of the individual from La Fage - Aven II to those of different recent and fossil specimens of wolverines and badgers.

présence, dans les loci $\mathrm{F}$ et $\mathrm{G}$ (au départ de la galerie nord, à une dizaine de mètres de profondeur) d'un abondant matériel osseux de cheval rapporté à Equus caballus cf. steinheimensis qui est la même sous-espèce que celle décrite dans l'Aven I (Bouchud 1972).

Le cheval presque complet recueilli dans le locus $\mathrm{D}$, à six mètres de profondeur, a été déterminé comme étant Equus caballus cf. piveteaui. En effet, toujours selon J. Bouchud (1978), " la forte taille de ses dents extrêmement hypsodontes et porteuses de caractères évolués l'écarte complètement du cheval de Steinheim ». C'est un cheval qui date de la fin du stade isotopique 6 (cf. notamment Delpech et Prat 1995 ; Langlois 2005) ; sa présence dans l'Aven II indique que les dépôts, s'ils sont pour une part aussi anciens que ceux de l'Aven I, sont en partie plus récents, datant de la fin du stade isotopique 6 . Recueillie deux mètres plus haut dans la stratigraphie, l'ulna de glouton du locus $\mathrm{B}$ pourrait être encore plus récente.

On ne trouve actuellement le glouton que dans les régions arctiques et subarctiques d'Amérique du Nord et d'Eurasie (complexe de faune mongolo-sibérienne, Schilling, Singer et Diller 1986 ; Prescott et Richard 1996 ; Aulagnier et al. 2008). Selon différents auteurs, l'adaptation du glouton au climat froid ne remonterait pas au-delà du Riss (Stehlin 1933 ; Piveteau 1961 ; Bonifay 1966) et pourrait donc être contemporaine des dépôts de l'Aven II de La Fage. D'autres en revanche considèrent que ce représentant des Mustélidés a toujours vécu sous un climat froid (Stehlin 1933 ; Kurtén 1968 ; Bonifay 1971 ; Griggo 1996), même si les conditions auraient pu être moins extrêmes qu'elles ne le sont dans son aire de répartition actuelle (Bonifay 1971; Argant 1991 ; Nowak 1991).

II préfère la toundra en été, bien qu'on puisse également le trouver dans les forêts et les reliefs rocheux escarpés. En hiver, il privilégie la forêt et la montagne. Son territoire couvre une surface allant de 100 à $2000 \mathrm{~km}^{2}$ (Schilling et al. 1986 ; Prescott \& Richard 1996 ; Aulagnier et al. 2008), ce qui signifie qu'il ne sera pas nécessairement représentatif de l'environnement immédiat du gisement. La conformation géographique et géomorphologique du site et de la région environnante est effectivement propice à l'existence d'un paysage « en mosaïque » (MourerChauviré et al. 1975) qui aurait procuré d'excellentes conditions de vie à des faunes a priori très différentes et d'origines diverses. Cette conformation du paysage est attestée pour le gisement voisin de La Fage-Aven I (Chaline 1975 ; Mourer-Chauviré et al. 1975), où une 
hémimandibule de Gulo gulo est mentionnée et décrite par M. Hugueney (1975). Nous pouvons probablement étendre cette réflexion à l'Aven II.

Quoi qu'il en soit, on ne peut raisonnablement admettre que le glouton ait choisi les régions à climat tempéré comme cadre de vie. II a vécu dans la partie septentrionale du Causse de Martel alors que les conditions étaient sans doute beaucoup plus froides qu'aujourd'hui. Cette avancée vers le sud de son aire de répartition s'est produite au moins à deux reprises puisque deux sujets sont représentés à La Fage, un dans les dépôts de l'Aven I qui dateraient du stade isotopique 8 ou 7 et un dans ceux de l'Aven II qui dateraient de la fin du stade isotopique 6 ou d'un stade plus récent. Précisons que, du point de vue du climat, la fin du stade isotopique 6 se caractérise par un passage rapide de conditions très rigoureuses («maximum glaciaire du stade 6») à des conditions presque tempérées annonçant la mise en place du sous-stade $5 \mathrm{e}$ («interglaciaire Riss-Würm») et l'on sait que, lors des stades suivants, des conditions climatiques sévères se sont plusieurs fois mises en place ; alors les zones boréales sont devenues des déserts froids et les aires de répartition animale se sont déplacées vers des zones aux conditions moins extrêmes (cf. Delpech 1999 et 2003 pour des précisions sur les mouvements de faune en Europe au Paléolithique supérieur). De nombreux gisements européens ont livré des restes de glouton (Bégouën et Koby 1950 ; Altuna 1963, 1972 ; Martin 1968 ; Bonifay 1971 ; Hugueney 1975 in Mourer-Chauviré et al. 1975 ; Guérin 1980 ; Clot 1982 ; Clot et Duranthon 1990 ; Argant 1991 ; Döppes 2001a, 2001b ; Mallye et Guérin 2002 ; Alvarez-Lao et Garcia 2010 ; Diedrich et Copeland 2010 ; Miracle et al. 2010). Nous n'en donnerons pas ici une liste exhaustive d'autant que leur âge, comme à La Fage II, ne peut toujours être précisé. Cependant, si l'on pointe leur situation géographique sur la carte de l'Europe, on met en évidence l'importance des déplacements qu'a subi l'aire de répartition du glouton sans doute plusieurs fois, comme nous l'avons écrit plus haut. Certains sites, comme la Grotte de Lezetxiki en Espagne et les grottes de Grimaldi en Italie, montrent que sa bordure méridionale a atteint sinon dépassé le $43^{\circ}$ parallèle (fig. 6).

\section{5 - Discussion et conclusion}

La morphologie de cet os chez le blaireau et le glouton étant proche, il est tout à fait compréhensible que ce fossile ait d'abord été déterminé comme étant un os de blaireau, $a$ fortiori puisqu'il s'agit d'un reste fragmentaire. C'est pourquoi, outre la détermination morphologique par anatomie comparée, une analyse des proportions était souhaitable afin d'appuyer cette argumentation.

Dans ce but, c'est la méthode du LSI qui fut retenue car l'échantillon ne se prête pas vraiment aux méthodes plus récentes de Variability Size Index (Uerpmann 1982 ; Escarguel 2008). Le diagramme obtenu permet de dissiper le doute quant à l'attribution du reste en montrant les tendances générales suivies par l'ensemble des mesures. Le sexe des individus est rarement déterminé, mais nous pouvons considérer que des membres des deux sexes sont représentés. L'ulna ne permet pas à elle seule de définir le sexe de l'individu.

Le DDP au niveau du processus styloïde du spécimen moins important que la moyenne des gloutons actuels pourrait être une simple variation individuelle, une caractéristique particulière de la population à laquelle il appartenait ou encore une adaptation écologique. On ne peut évidemment pas se prononcer à ce sujet sur la base d'un seul reste.

La petite taille de ce spécimen pourrait correspondre à une adaptation au froid, comme le suggèrent les règles de Bergmann (1847) et d'Allen (1877). Cependant nous ne pouvons conclure sur ce point à l'aide de ce seul reste ; un os ne représentant pas une variabilité de population. De plus M.F. Bonifay (1971) suggère que la taille du glouton n'aurait en aucun cas évolué de manière linéaire, mais au contraire aurait varié plusieurs fois tout au long du Quaternaire. Cela pourrait aussi être lié à l'abondance des proies et/ou à la présence de compétiteurs dans le milieu (cf. Boudadi-Maligne 2010). Enfin, cela pourrait être dû à une simple variabilité individuelle ou populationnelle, ou encore au sexe de l'individu. Nous ne nous prononcerons pas sur la seule base de ce fragment osseux.

La présence de cet ulna dans ce gisement est importante, car les fossiles de glouton restent rares, et s'inscrit dans les déplacements de l'aire de répartition de l'espèce en fonction des fluctuations climatiques du Pléistocène. Les avens de La Fage font ainsi partie des quelques sites à former la frange la plus méridionale où des gloutons ont été découverts en Europe.

\section{Remerciements}

Nous tenons à exprimer notre reconnaissance aux équipes du Museum National d'Histoire Naturelle de Paris et du Centre de Conservation et d'Étude des Collections de Lyon pour l'accueil et l'accès au matériel qu'ils nous ont accordés et leur disponibilité, en particulier à Céline Bens, Didier Berthet et François Vigouroux.

Notre gratitude va également à Nicolas Boulbes, Françoise Delpech, Jean-Baptiste Mallye et Anne-Marie Moigne, ainsi qu'aux rapporteurs de l'article pour leurs précieux conseils et les discussions qui ont permis de faire avancer le propos. Nous remercions aussi très chaleureusement Christelle Milizia pour le dessin du schéma de mesure de l'ulna et Svetlana Pleshakova pour son expertise de l'anglais. Notre plus profonde gratitude va également à Christine Boussat pour sa disponibilité et son expertise aimable et précieuse. 


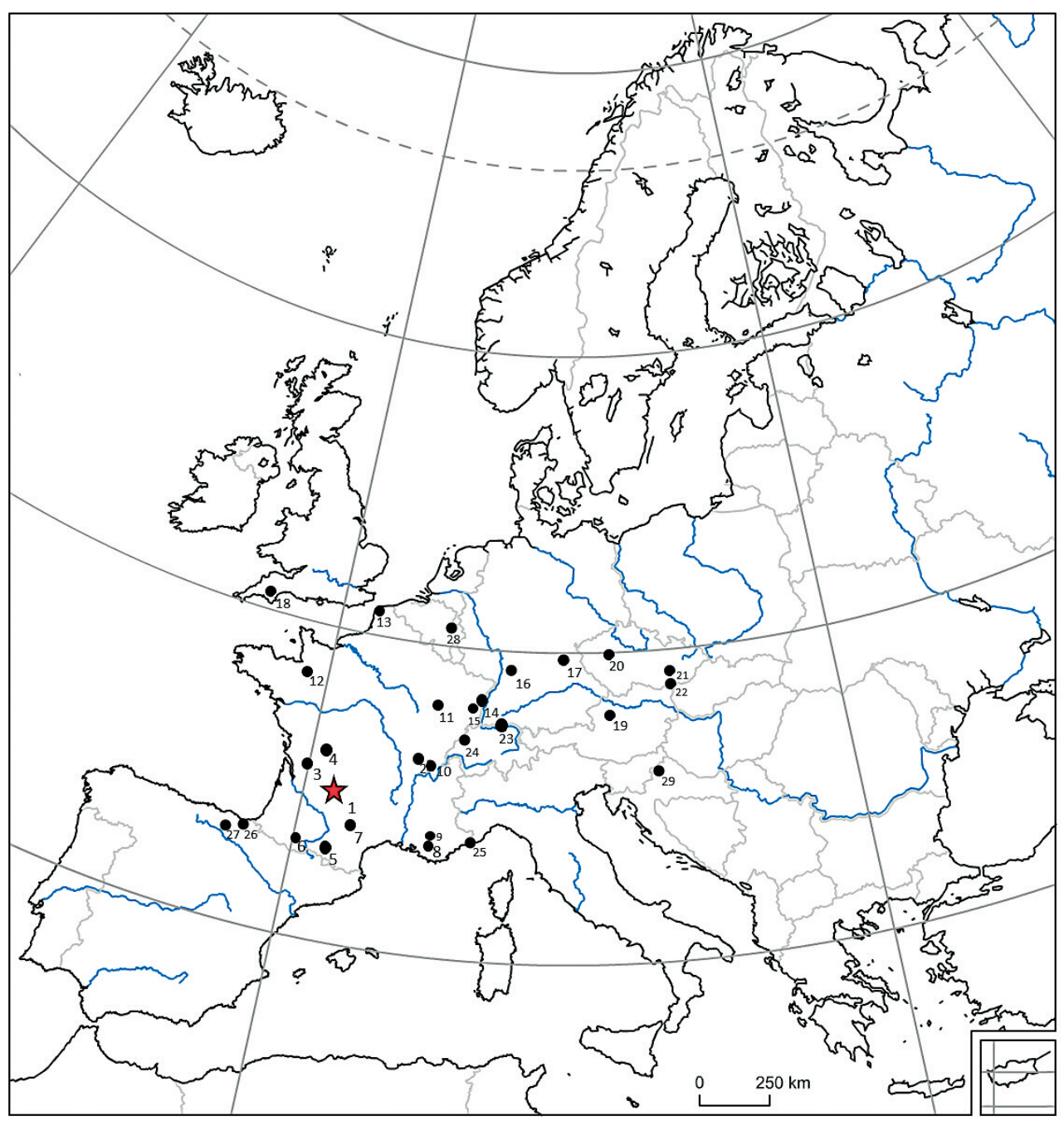

Figure 6 - Carte indiquant la position de différents sites européens du Pléistocène où la présence de glouton est attestée. 1 : Abîmes de La Fage et grotte de Jaurens. 2 : Verzé-Verchizeuil et Solutré. 3 : Grotte d'Artenac. 4 : Vilhonneur. 5 : Grottes Bernard/du Fustié, de l'Herm et des Trois-Frères. 6: Grotte de Labastide. 7 : Aven de Bouxès. 8 : Grotte de l'Adaouste. 9: La Valescure. 10 : Abri Gay et Villereversure. 11 : Grotte de Fouvent/Abri Cuvier. 12 : La Pointe du Bois. 13 : Grotte de RinxentHydrequent. 14 : Lingolsheim. 15 : Voegtlinshoffen/Vöklinshoffen. 16 : Mosbach. 17 : Grotte de Vogelherd. 18 : Grotte de Tornewton. 19: Grotte de Salzofen. 20 : Grottes de Srbsko, Chlupáčova Sluj et Praha-Podbaba. 21 : Grottes de Kůlna a Křižovy, Sloup et Výpustek. 22 : Dolni Věstonice. 23 : Grotte du Kesslerloch et Abri de Schweizerbild. 24 : Grotte de Cotencher. 25 : Grotte de Grimaldi. 26 : Grotte de Lezetxiki. 27 : Grotte de Mairuelegorreta. 28 : Trou du Moulin. 29 : Grottes de Velika Pećina et Vindija.

Figure 6 - Map showing the position of different european sites of Pleistocene where wolverine presence is attested. 1: La Fage and Jaurens caves. 2: Verzé-Verchizeuil and Solutré. 3: Cave of Artenac. 4: Vilhonneur. 5: Bernard/Fustié, Herm and Trois-Frères caves. 6: Cave of Labastide. 7: Aven of Bouxès. 8: Cave of Adaouste. 9: La Valescure. 10: Abri Gay and Villereversure. 11: Cave of Fouvent/Abri Cuvier. 12: La Pointe du Bois. 13: Cave of Rinxent-Hydrequent. 14: Lingolsheim. 15: Voegtlinshoffen/ Vöklinshoffen. 16: Mosbach. 17: Vogelherd cave. 18: Tornewton cave. 19: Salzofen cave. 20: PrahaPodbaba, Srbsko and Chlupáčova Sluj caves. 21: Kưlna a Křižovy, Sloup and Výpustek caves. 22: Dolni Věstonice. 23: Kesslerloch and Schweizerbild caves. 24: Cotencher cave. 25: Cave of Grimaldi. 26: Cave of Lezetxiki. 27: Mairuelegorreta cave. 28: Trou du Moulin. 29: Velika Pećina and Vindija caves. 


\section{Références bibliographiques}

ALLEN J.A. 1877 - The influence of Physical conditions in the genesis of species. Radical Review, 1: p. 108-140.

ALTUNA J. 1963 - Primer hallazgo de glotón (Gulo gulo L.) en la Península Ibérica. Munibe, 15, p. 128.

ALTUNA J. 1972 - Fauna de Mamíferos de los Yacimientos Prehistóricos de Guipúzcoa. Munibe, 24 (1-4), 1-464.

ÁLVAREZ-LAO D.J. et GARCÍA N. 2010 - Chronological distribution of Pleistocene cold-adapted large mammal faunas in the Iberian Peninsula. Quaternary International 212, p. $120-128$

ARGANT A. 1991 - Carnivores Quaternaires de Bourgogne. Documents des laboratoires de géologie, Lyon 1, $115: 301 \mathrm{p}$.

AULAGNIER S., HAFFNER P., MITCHELL-JONES A.J., MOUTOU F. et ZIMA J. 2008 - Guide des Mammifères d'Europe, d'Afrique du Nord et du Moyen-Orient, Les Guides du Naturaliste, Ed. Delachaux \& Niestlé: p. $112-113$

BARONE R. 1976 - Anatomie comparée des mammifères domestiques. Ed. Vigot Frères. t.1, Ostéologie, fasc. 1 et 2. $2^{\mathrm{e}}$ édition.

BEGOUËN L. et KOBY F.-E. 1950 - Le Crâne de Glouton de la Caverne des Trois-Frères (Ariège), Préhistoire ariégeoise, p. 49-68.

BERGMANN C. 1847 - Über die Verhältnisse des Wärmekönomie des Thiere zu ihrer Grösse. Göttinger Studine, 1, p. 595-708.

BONIFAY M.F. 1966 - La famille des Mustélidés. p. 381396 In R. Lavocat Atlas de Préhistoire - Faunes et flores préhistoriques de l'Europe Occidentale. Chapitre VII. Ed N. Boubée et Cie, Paris.

BONIFAY M.F. 1971 - Carnivores quaternaires du Sud-Est de la France. Mém. Mus. Nat. Hist. nat. Paris, Paris. 377 p.

BOUCHUD J. 1972 - Les grands herbivores rissiens du gisement paléontologique de La Fage. Nouv. Arch. Mus. Hist. nat. Lyon, fasc. 10, p. 33-59, fig. 15-20, tabl. 1-37, pl. 10-13.

BOUCHUD J. 1978 - Les grands herbivores rissiens de l'Aven II des Abîmes de La Fage, à Noailles (Corrèze). Nouv. Arch. Mus. Hist. nat. Lyon, fasc. 16, p. 9-39, 26 tabl., 4 pl.

BOUDADI-MALIGNE M. 2010 - Les Canis pleistocènes du Sud de la France : Approche Biosystématique, Evolutive et Biochronologique. Thèse de Doctorat de l'Université de Bordeaux I. 446 p. Bordeaux.

BOURGEOIS G. 2012 - Des blaireaux en milieu périurbain au IV s. In : Quatre puits de l'agglomération routière gallo- romaine d'Ambrussum (Villetelle, Hérault). Sous la direction de Jean-Luc FICHES. Revue Archéologique de Narbonnaise, supplément 42, chapitre 18, p.327-333. Éditions de l'Association de la Revue archéologique de Narbonnaise Montpellier-Lattes.

CHALINE J. 1975 - Les rongeurs, l'âge et la chronologie climatique du remplissage de l'Aven I de la Fage (Corrèze). Nouv Arch Mus Hist nat Lyon, fasc. 13, p. 113-117, fig. 36.

CLOT A. 1982 - Le Glouton (Gulo gulo L., Mustelidae, Carnivora) de Labastide (Hautes-Pyrénées), Bull. Sté Hist. Nat. Toulouse, 118, p.101-109.

CLOT A. et DURANTHON F. 1990 - Les mammifères fossiles du Quaternaire dans les Pyrénées, Muséum d'Histoire Naturelle de Toulouse, p. 104-112.

DELPECH F. 1999 - Biomasse d'Ongulés au paléolithique et inférences démographiques. Paleo, $\mathrm{n}^{\circ}$ 11, p.19-42, 10 fig., 4 tabl.

DELPECH F. 2003 - L'environnement animal des européens au Paléolithique supérieur. In R. Desbrosse et A. Thévenin (eds) : Préhistoire de l'Europe. Des origines à l'Age du Bronze. Actes des Congrès nationaux des Sociétés historiques et scientifiques. $125^{\mathrm{e}}$, Lille 2000, Editions du C.T.H.S., Paris, p. 271-289.

DELPECH F. et PRAT F. 1995 - Nouvelles observations sur les faunes acheuléennes de Combe-Grenal (Domme, Dordogne). Paleo, n 7, p. 123-137.

DIEDRICH C.G. et COPELAND J.P. 2010 - Upper Pleistocene Gulo gulo (Linné, 1758) remains from the Srbsko Chlum-Komin hyena den cave in the Bohemian Karst, Czech Republic, with comparisons to contemporary wolverines. Journal of Cave and Karst Studies, v. 72, $\mathrm{n}^{\circ} .2$, p. 122-127.

DÖPPES D. 2001a - Gulo gulo (Mustelidae, Mammalia) im Jungpleitozän Mitteleuropas, Beitr. Paläont. Österr. 26, $95 \mathrm{p}$.

DÖPPES D. 2001b - The wolverine (Gulo gulo L.) in Spain - one of the south most spreading during the Pleistocene. Cadernos Lab. Xeolóxico de Laxe Coruña. 2001. Vol. 26, p. 479-484. ISSN: 0213-4497.

ESCARGUEL G. 2008 - Macroécologie en terme profond: motifs, rythmes et modalités des changements de biodiversité à l'échelle des temps géologiques. Mémoire d'Habilitation à Diriger des Recherches, Université Claude Bernard, Lyon 1, $430 \mathrm{p}$.

GRIGGO C. 1996 - Etablissement de courbes climatiques quantifiées à partir des communautés animales pléistocènes suivi d'une application aux gisements de l'abri Suard (Charente) et la grotte de Bois-Ragot (Vienne). In : Paleo ${ }^{\circ}$ 8, p. 81-97.

GUÉRIN C. 1980 - Les rhinocéros (Mammalia, Perissodactyla) du Miocène terminal au Pléistocène 
supérieur en Europe occidentale. Comparaison avec les espèces actuelles, Documents des laboratoires de géologie de Lyon, 79, fasc. 1,2,3, $1185 \mathrm{p}$.

GUÉRIN C. et PHILIPPE M. 1971 - Les gisements de vertébrés pléistocènes du causse de Martel. Bull. Soc. Sc., hist. archéol. Corrèze, Brive, t. 93, p. 31-46, 4 fig.

HUGUENEY M. 1975 - Les Mustélidés (Mammalia, Carnivora) du gisement Pléistocène moyen de La Fage (Corrèze). Nouv Arch Mus Hist nat Lyon, fasc. 13, p. 29-46, fig. 13-18, pl.1.

KURTÉN B. 1968 - Pleistocene Mammals of Europe, London. 317 p.

LANGLOIS A. 2005 - Le Cheval du gisement pléistocène moyen de La Micoque (Les Eyzies-de-Tayac, Dordogne) : Equus mosbachensis micoquii nov. ssp., Paleo, $\mathrm{n}^{\circ} 17$, p. $73-110$

LAVOCAT R. 1966 - Atlas de préhistoire. Ed. N. Boubée et Cie, t. 3, Faunes et Flores préhistoriques de l'Europe occidentale, 486 p., nbses pl. et fig.

MALLYE J.-B. et GUÉRIN C. 2002 - Le gisement Pléistocène supérieur de la grotte de Jaurens à Nespouls, Corrèze, France : les Mustelidae (Mammalia, Carnivora). Cahiers scientifiques du Musém d'Histoire naturelle de Lyon, fasc. 1, p. 15-53, 7 fig., 17 tabl., 2 pl.

MARTIN R. 1967 - Le glouton de Villereversure. Doc. Lab. Géol. Fac. Sci. Lyon, n 21, p. 71-110.

MARTIN R. 1968 - Les Mammifères fossiles du gisement quaternaire de Villereversure (Ain). Etude des Carnivores, des Cervidés et des Equidés. Doc. Lab. Géol. Fac. Sci. Lyon, $\mathrm{n}^{\circ}$ 27, 153 p., 41 fig., 36 tabl.

MIRACLE P.T., LENARDIC J.M. et BARJKOVIC D. 2010 Last glacial climates, "Refugia », and faunal change in Southeastern Europe: Mammalian assemblages from Veternica, Velika pecina, and Vindija caves (Croatia). Quaternary International 212, p. 137-148.

MOURER-CHAUVIRÉ C., avec la collaboration de BALESIO R., BEDEN M., BOUCHUD J., CHALINE J., DAVID L., GUERIN C., HUGUENEY M., JAMMOT D., MEIN P., RAGE J.C. et VILAIN R. 1975 - Conclusions générales sur les faunes de l'Aven I des Abîmes de La Fage (Corrèze). Nouv. Arch. Mus Hist. nat. Lyon, fasc. 13, p. 123-129.
MOURER-CHAUVIRÉ C., PHILIPPE M., QUINIF Y., CHALINE J., DEBARD E., GUERIN C. \& HUGUENEY M. 2003 - Position of the palaeontological site Aven I des Abîmes de La Fage, at Noailles (Corrèze, France), in the European Pleistocene chronology. Boreas, Vol. 32, p. 521531. Oslo. ISSN 0300-9483.

NOWAK R.M. 1991 - Walker's Mammals of the World, fifth ed. Johns Hopkins University Press, Baltimore.

PALES L. et LAMBERT C. 1971 - Atlas ostéologique pour servir à l'identification des mammifères du Quaternaire. Ed. du C.N.R.S., t.1, Les membres. p. 6, 10.

PHILIPPE M. 1978 - Présentation du gisement pléistocène moyen de l'Aven II des Abîmes de La Fage, à Noailles (Corrèze). Nouv. Arch. Mus. Hist. nat. Lyon, fasc. 16, p. 3-8, 2 fig.

PHILIPPE M., MOURER-CHAUVIRÉ C. et ÉVIN J. 1981 Les gisements paléontologiques quaternaires des causses de Martel et de Gramat (Corrèze et Lot) : faunes et chronologie. Nouv. Arch. Mus. Hist. nat. Lyon, fasc. 18, suppl. p. 57-67, 1 fig.

PIVETEAU J. 1961 - Traité de Paléontologie. Ed. Masson. t. 6, v. 1, Les Carnivores.

PRESCOTT J. et RICHARD P. 1996 - Mammifères du Québec et de l'Est du Canada, Waterloo (Québec), 399 p.

SCHILLING D., SINGER D. et DILLER H. 1986 - Guide des Mammifères d'Europe, Neuchâtel, 280 p.

SIMPSON G. G. 1941 - Large Pleistocene Felines of North America. American Museum Novitates, 1136: p.1-27.

STELHIN H.G. 1933 - La grotte de Cotencher, station moustérienne. In : DUBOIS A. et STELHIN H.G. Mém. Soc. Paléont. suisse, Bâle, vol. 52-53, 292 p., 37 fig., 15 pl.

UERPMANN H.-P. 1982 - Faunal Remains from Shams edDin Tannira, a Halafian Site in Northern Syria. Berytus Archaeological Studies Beyrouth, vol. 30, p. 3-52.

VON DEN DRIESCH A. 1976 - A guide to the measurement of animal bones from archaeological sites. Peabody Museum bulletins. Bulletin 1. Peabody Museum of archaeology and ethnology. Harvard University. 
